\title{
CONE INVARIANCE AND SQUEEZING PROPERTIES FOR INERTIAL MANIFOLDS FOR NONAUTONOMOUS EVOLUTION EQUATIONS
}

\author{
NORBERT KOKSCH \\ Department of Mathematics, Technical University Dresden \\ 01062 Dresden, Germany \\ E-mail:koksch@math.tu-dresden.de \\ STEFAN SIEGMUND \\ Department of Mathematics, University of California \\ Berkeley, CA 94720, USA \\ E-mail:siegmund@math.berkeley.edu
}

\begin{abstract}
In this paper we summarize an abstract approach to inertial manifolds for nonautonomous dynamical systems. Our result on the existence of inertial manifolds requires only two geometrical assumptions, called cone invariance and squeezing property, and some additional technical assumptions like boundedness or smoothing properties. We apply this result to processes (two-parameter semiflows) generated by nonautonomous semilinear parabolic evolution equations.
\end{abstract}

1. Introduction. Let us consider a nonlinear evolution equation of the form

$$
\dot{u}+A u=f(u)
$$

in a Banach space $\mathcal{X}$, where $A$ is a linear sectorial operator with compact resolvent and $f$ is a nonlinear function. Such an evolution equation may be an ordinary differential equation $\left(\mathcal{X}=\mathbb{R}^{n}\right)$ or the abstract formulation of a semilinear parabolic differential equation with $\mathcal{X}$ as a suitable function space over the spatial domain. In the last case, $A$ corresponds to a linear differential operator and $f$ is a nonlinearity which may involve derivatives of lower order than $A$.

Inertial manifolds are positively invariant, exponentially attracting, finite dimensional Lipschitz manifolds. For an introduction see Sell and You [SY02]. They go back to R. Mañé, D. Henry, X. Mora, and D. A. Kamaev [Hen81, Mor83, Man77, Kam84] and were first introduced and studied by P. Constantin, C. Foias, B. Nicolaenko, G. R. Sell

2000 Mathematics Subject Classification: 34C30, 34D45, 34G20, 35B42, 37L25.

The paper is in final form and no version of it will be published elsewhere. 
and R. Temam [FST85, FNST85, CFNT86] for selfadjoint $A$. For the construction of inertial manifolds with $A$ being non-selfadjoint see for example [SY92] and [Tem97]. Inertial manifolds are generalizations of center-unstable manifolds and they are more convenient objects which capture the long-time behavior of dynamical systems. Usually an inertial manifold $\mathcal{M}$ is seeked as the graph of a sufficiently smooth function $m$ on $P \mathcal{X}$, i.e.

$$
\mathcal{M}=\operatorname{graph}(m):=\{x+m(x): x \in P \mathcal{X}\},
$$

where $P$ is a finite dimensional projector. The finite dimensionality and the exponential attracting property permit the reduction of the dynamics of the infinite or high dimensional equation to the dynamics of a finite or low dimensional ordinary differential equation

$$
\dot{x}+A x=P f(x+m(x)) \quad \text { in } P \mathcal{X}
$$

called inertial form system. A stronger reduction property is the exponential tracking property [FST89] or the asymptotical completeness property [CFNT89a, Rob96, Tem97]: Each trajectory of the evolution equation tends exponentially to a trajectory in the inertial manifold.

Thus, one can reduce (in some sense) an infinite-dimensional physical system to a system with a finite number of degrees of freedom and use known properties of ordinary differential equations in $\mathbb{R}^{n}$ for the qualitative analysis of the behavior of the solutions of the original equation for large time. Another advantage of studying the inertial form system is the reduction of the dimension of the original problem which could be important for the numerical analysis. In concrete applications, the $N$-dimensional projector $P$ is the projector onto the eigenspace spanned by the first $N$ Fourier modes of $L$, i.e. by eigenvectors of $A$ belonging to the set of the $N$ smallest eigenvalues of $A$. Hence the inertial form systems describes the dynamics of the slow modes. For applications of the concept of inertial manifolds see for example [HR92, RB95, SK95, BH96, MSZ00].

There are a few ways of constructing an inertial manifold. Most of them are generalizations of methods developed for the construction of unstable, stable, center-unstable or center manifolds for ordinary differential equations:

The Lyapunov-Perron method has been developed by A. M. Lyapunov [Lya47, Lya92] and O. Perron [Per28, Per29, Per30] for the proof of the existence of stable and unstable manifolds of hyperbolic equilibrium points. In the context of ordinary differential equations, it deals with the integral equation formulation of the differential equation and constructs the invariant manifold as a fixed point of an operator that is derived from this integral equation. This method has been used in many different situations. The book of J. K. Hale [Hal80] may serve as a good reference. In the infinite dimensional setting, the method is used by D. Henry [Hen81] to prove the existence of stable, unstable and center manifolds for semilinear parabolic equations. In [FST88, Tem88, CFNT89b, FST89, DG91], it has been adapted for the construction of inertial manifolds. At the moment, the Lyapunov-Perron method is the most common method for inertial manifolds.

Hadamard's method [Had01], also called graph transformation method, has been developed to prove the existence of stable and unstable manifolds of fixed points of diffeomorphisms. The graph transformation method is more geometrical in nature than 
the Lyapunov-Perron method. In presence of a hyperbolic fixed point, the stable and unstable manifolds are constructed as graphs over the linearized stable and unstable subspaces. Mallet-Paret and Sell [MPS88] were the first who used Hadamard's method for the construction of inertial manifolds, see also [BJ89, Rob93, Rob01]. A review on different construction methods (including Hadamard's method) for inertial manifolds can be found in [LS89, Nin93].

It appears that independent of the method used a special condition is utilized predominantly of the form

$$
\Lambda_{2}-\Lambda_{1}>C L\left(\Lambda_{1}^{\nu}+\Lambda_{2}^{\nu}\right)
$$

usually referred to as a spectral gap condition. This spectral gap condition assumes that the spectrum $\sigma(A)$ of the linear part $A$ is split into a finite set of eigenvalues with real part less or equal to $\Lambda_{1}$ and the remaining set of eigenvalues with real part greater than or equal to $\Lambda_{2}$, and that the difference of $\Lambda_{2}$ and $\Lambda_{1}$ is large enough to majorize $C L\left(\Lambda_{1}^{\nu}+\Lambda_{2}^{\nu}\right)$, where $L$ is a global Lipschitz constant for $f, \nu \in[0,1[$ is an additional parameter depending on $f$, and $C$ is a technical constant depending on the particular proof used. By utilizing indefinite quadratic forms, A. V. Romanov [Rom94] found a spectral gap condition with $C=1$ and he showed that this condition is sharp.

The notion of inertial manifolds mentioned above is translated and extended to more general classes of differential equations like nonautonomous differential equations [GV97, WF97, LL99], retarded parabolic differential equations [TY94, BdMCR98], or differential equations with random or stochastic perturbations [Chu95, BF95, CL99, CS01, DLS01]. Here spectral gap conditions are also utilized, where the special form of this condition may depend on the assumptions on the nonlinearity and, in particular, on the delay.

Since the spectral gap condition is the most restrictive condition with regard to applications, it is of interest to weaken this condition or even to find sharp conditions. One way to weaken the assumptions is to work out the essential properties of the semiflow, the process (two-parameter semiflow), or the skew product semiflow which are used for the construction of the inertial manifold. Such geometrical properties are the cone invariance and the squeezing property. The cone invariance property describes the fact that the difference of two trajectories cannot leave a certain cone. This property is mainly used to prove the existence of the manifold. Robinson [Rob93] has shown that the cone invariance property is sufficient to prove the existence of an inertial Lipschitz manifold. The squeezing property requires that the difference of each pair of solutions decays exponentially as long as the difference is outside of a possibly another cone; it is used to prove the attraction properties. A combination of cone invariance and squeezing properties for evolution equations, sometimes called strong squeezing property, was first introduced for the Kuramoto-Sivashinsky equations in [FNST85, FNST88], an abstract version of it was developed in [FST89], another formulation of it can be found for example in [Tem88, FST88, CFNT89a, Rob93, JT96]. Essentially, a strong squeezing property states that if the difference of two solutions of the evolution equation belongs to a special cone then it remains in the cone for all further times (that is the cone invariance property); otherwise the distance between the solutions decays exponentially (that is the squeezing property). 
In [KS02], the notion of a nonautonomous inertial manifold for nonautonomous dynamical systems is introduced. Nonautonomous dynamical systems are generalizations of semiflows, processes and skew product semiflows. Moreover, in [KS02, KS01], the existence of pullback attracting inertial manifolds is shown assuming appropriate cone invariance and squeezing properties and assuming additional technical assumptions which guarantee that the function $m$ generating the manifold can be constructed in a space of bounded Lipschitz functions, see [KS02] assuming a boundedness and coercivity property, or in a space of linearly bounded Lipschitz functions, see [KS01] assuming a stationarity and strong coercivity property. Moreover, in [KS02] we use a cone invariance property which uses cones which may vary with the base variable, and the constants in the squeezing and boundedness properties are required to be uniform only on orbits of the driving system and not on the whole base space.

In this paper we summarize the main results of [KS02, KS01] for processes generated by nonautonomous evolution equations.

2. Inertial manifolds for nonautonomous dynamical systems. Let $\mathcal{X}$ be a Banach space.

Definition 2.1. A nonautonomous dynamical system (NDS) on $\mathcal{X}$ is a cocycle $\varphi$ over a driving system $\theta$ on a set $\mathcal{B}$, i.e.

(i) $\theta: \mathbb{R} \times \mathcal{B} \rightarrow \mathcal{B}$ is a dynamical system, i.e. the family $\theta(t, \cdot)=\theta(t): \mathcal{B} \rightarrow \mathcal{B}$ of self-mappings of $\mathcal{B}$ satisfies the group property

$$
\theta(0)=\operatorname{id}_{\mathcal{B}}, \quad \theta(t+s)=\theta(t) \circ \theta(s)
$$

for all $t, s \in \mathbb{R}$.

(ii) $\varphi: \mathbb{R}_{\geq 0} \times \mathcal{B} \times \mathcal{X} \rightarrow \mathcal{X}$ is a cocycle, i.e. the family $\varphi(t, b, \cdot)=\varphi(t, b): \mathcal{X} \rightarrow \mathcal{X}$ of self-mappings of $\mathcal{X}$ satisfies the cocycle property

$$
\varphi(0, b)=\operatorname{id}_{\mathcal{X}}, \quad \varphi(t+s, b)=\varphi(t, \theta(s) b) \circ \varphi(s, b)
$$

for all $t, s \geq 0$ and $b \in \mathcal{B}$. Moreover $(t, x) \mapsto \varphi(t, b, x)$ is continuous.

REMARK 2.2.

(i) The set $\mathcal{B}$ is called base and in applications it has additional structure, e.g. it is a probability space, a topological space or a compact group and the driving system has additional regularity, e.g. it is ergodic or continuous.

(ii) The pair of mappings

$$
(\theta, \varphi): \mathbb{R}_{\geq 0} \times \mathcal{B} \times \mathcal{X} \rightarrow \mathcal{B} \times \mathcal{X}, \quad(t, b, x) \mapsto(\theta(t, b), \varphi(t, b, x))
$$

is a special semi-dynamical system a so-called skew product flow (usually one requires additionally that $(\theta, \varphi)$ is continuous). If $\mathcal{B}=\{b\}$ consists of one point then the cocycle $\varphi$ is a semi-dynamical system.

(iii) We use the abbreviations

$$
\theta_{t} b:=\theta(t) b:=\theta(t, b), \quad \varphi(t, b) x:=\varphi(t, b, x) .
$$

We also say that $\varphi$ is an NDS to abbreviate the situation of Definition 2.1. 
The solutions of a nonautonomous evolution equation in $\mathcal{X}$ will not generate a semiflow but a so-called process (Dafermos [Daf71]) (or two-parameter semi-flow).

Definition 2.3. A process or two-parameter semi-flow $\mu$ on $\mathcal{X}$ is a continuous mapping

$$
\{(t, s, x) \in \mathbb{R} \times \mathbb{R} \times \mathcal{X}: t \geq s\} \ni(t, s, x) \mapsto \mu(t, s, x) \in \mathcal{X}
$$

which satisfies

(i) $\mu(s, s, \cdot)=\operatorname{id} \mathcal{X}$ for $s \in \mathbb{R}$;

(ii) the two-parameter semi-flow property for $t \geq \tau \geq s, x \in \mathcal{X}$, i.e.

$$
\mu(t, \tau, \mu(\tau, s, x))=\mu(t, s, x) .
$$

The next lemma explains how a process defines an NDS and how one can translate properties from the language of nonautonomous dynamical systems to the language of processes or vice versa.

Lemma 2.4. Suppose that $\mu$ is a process. Then $\varphi: \mathbb{R}_{\geq 0} \times \mathcal{B} \times \mathcal{X} \rightarrow \mathcal{X}$,

$$
\varphi(t, b) x=\mu(t+b, b, x)
$$

is an $N D S$ with base $\mathcal{B}=\mathbb{R}$ and driving system $\theta: \mathbb{R} \times \mathcal{B} \rightarrow \mathcal{B}$,

$$
\theta(t) b=t+b .
$$

Moreover, for $t \geq s$ and $x \in \mathcal{X}$ the relation $\mu(t, s, x)=\varphi(t-s, s) x$ holds.

Definition 2.5. A family $\mathcal{M}=(\mathcal{M}(b))_{b \in \mathcal{B}}$ of non-empty sets $\mathcal{M}(b) \subset \mathcal{X}$ is called a nonautonomous set and $\mathcal{M}(b)$ is called the $b$-fiber of $\mathcal{M}$ or the fiber of $\mathcal{M}$ over $b$. We say that $\mathcal{M}$ is closed, open, bounded, or compact, if every fiber has the corresponding property. For notational convenience we use the identification $\mathcal{M} \simeq\{(b, x): b \in \mathcal{B}$, $x \in \mathcal{M}(b)\} \subset \mathcal{B} \times \mathcal{X}$.

Definition 2.6. A nonautonomous set $\mathcal{M}$ is called forward invariant under the $\operatorname{NDS} \varphi$ if $\varphi(t, b) \mathcal{M}(b) \subset \mathcal{M}\left(\theta_{t} b\right)$ for $t \geq 0$ and $b \in \mathcal{B}$. It is called invariant if $\varphi(t, b) \mathcal{M}(b)=$ $\mathcal{M}\left(\theta_{t} b\right)$ for $t \geq 0$ and $b \in \mathcal{B}$.

Definition 2.7. Let $\varphi$ be an NDS. Then a nonautonomous set $\mathcal{M}$ is called (nonautonomous) inertial manifold if

(i) every fiber $\mathcal{M}(b)$ is a finite-dimensional Lipschitz manifold in $\mathcal{X}$ of dimension $N$ for an $N \in \mathbb{N}$;

(ii) $\mathcal{M}$ is invariant;

(iii) $\mathcal{M}$ is exponentially attracting, i.e. there exists a positive constant $\eta$ such that for every $b \in \mathcal{B}$ and $x \in \mathcal{M}(b)$ there exists an $x^{\prime} \in \mathcal{M}(b)$ with

$$
\left\|\varphi(t, b) x-\varphi(t, b) x^{\prime}\right\| \leq K \mathrm{e}^{-\eta t} \quad \text { for } t \geq 0 \text { and } b \in \mathcal{B}
$$

and a constant $K=K\left(b, x, x^{\prime}\right)>0$.

The property (iii) is also called exponential tracking property or asymptotic completeness property and $x^{\prime}$ or $\varphi(\cdot, b) x^{\prime}$ is said to be the asymptotic phase of $x$ or $\varphi(\cdot, b) x$, respectively. 
Recall that if $\mathcal{D}$ and $\mathcal{A}$ are nonempty closed sets in $\mathcal{X}$, the Hausdorff semi-metric $d(\mathcal{D} \mid \mathcal{A})$ is defined by

$$
d(\mathcal{D} \mid \mathcal{A}):=\sup _{x \in \mathcal{D}} d(x, \mathcal{A}), \quad d(x, \mathcal{A}):=\inf _{y \in \mathcal{A}} d(x, y)=\inf _{y \in \mathcal{A}}\|x-y\| .
$$

Now we want to generalize the attracting notion of dynamical systems to nonautonomous dynamical systems. A natural generalization of convergence to a nonautonomous set $\mathcal{A}$ seems to be the forwards running convergence defined by

$$
d\left(\varphi(t, b) x, \mathcal{A}\left(\theta_{t} b\right)\right) \rightarrow 0 \quad \text { for } t \rightarrow \infty .
$$

However, this does not ensure convergence to a specific component set $\mathcal{A}(b)$ for a fixed $b$. For that one needs to start "progressively earlier" at $\theta_{-t} b$ in order to "finish" at $b$. This leads to the concept of pullback convergence defined by

$$
d\left(\varphi\left(t, \theta_{-t} b\right) x, \mathcal{A}(b)\right) \rightarrow 0 \quad \text { for } t \rightarrow \infty .
$$

Definition 2.8. Let $\varphi$ be an NDS and $\mathcal{A}$ be a nonautonomous set. $\mathcal{A}$ is called (globally) pullback attracting if for every bounded set $\mathcal{D} \subset \mathcal{X}$ and $b \in \mathcal{B}$

$$
\lim _{t \rightarrow \infty} d\left(\varphi\left(t, \theta_{-t} b\right) \mathcal{D} \mid \mathcal{A}(b)\right)=0 \text {. }
$$

The concept of pullback convergence was introduced in the mid 1990s in the context of random dynamical systems (see Schmalfuss [Sch92], Crauel and Flandoli [CF94], Flandoli and Schmalfuss [FS96], and Crauel, Debussche and Flandoli [CDF97]) and has been used e.g. in numerical dynamics, see for example [KS97b, KS97a, KS98]. Note that a similar idea had already been used in the 1960s by Mark Krasnoselski [Kra68] to establish the existence of solutions that exist and remain bounded on the entire time set.

Now we define a handy notion (see Ludwig Arnold [Arn98, Definition 4.1.1(ii)]) excluding exponential growth of a function.

Definition 2.9. A function $R: \mathcal{B} \rightarrow] 0, \infty$ [ is called tempered from above if for every $b \in \mathcal{B}$

$$
\limsup _{t \rightarrow \pm \infty} \frac{1}{|t|} \log R\left(\theta_{t} b\right)=0
$$

Definition 2.10. Let $\varphi$ be an NDS. A family $\pi=(\pi(b))_{b \in \mathcal{B}}$ of projectors $\pi(b) \in$ $L(\mathcal{X}, \mathcal{X})$ in $\mathcal{X}$ is called nonautonomous projector.

(i) $\pi$ is called tempered from above if $b \mapsto\|\pi(b)\|$ is tempered from above.

(ii) $\pi$ is called $N$-dimensional for an $N \in \mathbb{N}$ if $\operatorname{dim} \operatorname{im} \pi(b)=N$ for every $b \in \mathcal{B}$.

Our goal is to construct an inertial manifold in $\mathcal{X}$. For this we will use some assumptions with respect to the norm of $\mathcal{X}$. In order to be more general, we will allow that some assumptions are required only with respect to the weaker norm of the larger space $\mathcal{Y}$, i.e., $(\mathcal{X}, \mathcal{Y})$ let be a pair of two Banach spaces such that $\mathcal{X}$ is continuously embedded in $\mathcal{Y}$,

$$
\mathcal{X} \hookrightarrow \mathcal{Y} .
$$

To compensate the different quality of the norms we need some smoothing action of the dynamical system. Note that in many cases one can use $\mathcal{X}=\mathcal{Y}$, and for a first reading it is good to assume $\mathcal{X}=\mathcal{Y}$. 
Let $\pi_{1}$ be an $N$-dimensional nonautonomous projector in $\mathcal{Y}$. We define the complementary projector

$$
\pi_{2}(b):=\operatorname{id} \mathcal{Y}-\pi_{1}(b) \quad \text { for } b \in \mathcal{B}
$$

Then

$$
\mathcal{X}_{1}(b):=\pi_{1}(b) \mathcal{X} \quad \text { and } \quad \mathcal{X}_{2}(b):=\pi_{2}(b) \mathcal{X}, \quad b \in \mathcal{B},
$$

define nonautonomous sets $\mathcal{X}_{i}$ consisting of complementary linear subspaces $\mathcal{X}_{i}(b)$ of $\mathcal{X}$, i.e. $\mathcal{X}_{1}(b) \oplus \mathcal{X}_{2}(b)=\mathcal{X}$. For this fact we also write $\mathcal{X}_{1} \oplus \mathcal{X}_{2}=\mathcal{B} \times \mathcal{X}$. Further let

$$
\mathcal{Y}_{1}(b):=\pi_{1}(b) \mathcal{Y} \quad \text { and } \quad \mathcal{Y}_{2}(b):=\pi_{2}(b) \mathcal{Y}, \quad b \in \mathcal{B} \text {. }
$$

We assume that

$$
\mathcal{X}_{1}(b)=\mathcal{Y}_{1}(b), \quad b \in B .
$$

We say that $\pi_{1}$ is tempered from above in $\mathcal{X}$ if the restriction $\left(\left.\pi_{1}(b)\right|_{\mathcal{X}}\right)_{b \in \mathcal{B}}$ of $\pi_{1}$ onto $\mathcal{X}$ is tempered from above.

We want to construct a nonautonomous inertial manifold

$$
\mathcal{M}=(\mathcal{M}(b))_{b \in \mathcal{B}}
$$

consisting of manifolds $\mathcal{M}(b) \subset \mathcal{X}$ which are trivial in the sense that each of them can be described by a single chart, i.e.

$$
\mathcal{M}(b)=\operatorname{graph}(m(b, \cdot)):=\left\{x_{1}+m\left(b, x_{1}\right): x_{1} \in \mathcal{X}_{1}(b)\right\}
$$

with $m(b, \cdot)=m(b): \mathcal{X}_{1}(b) \rightarrow \mathcal{X}_{2}(b)$.

For a positive constant $L$ we introduce the nonautonomous set

$$
\mathcal{C}_{L}:=\left\{(b, x) \in \mathcal{B} \times \mathcal{X}:\left\|\pi_{2}(b) x\right\|_{\mathcal{Y}} \leq L\left\|\pi_{1}(b) x\right\|_{\mathcal{Y}}\right\} .
$$

Since the fibers $\mathcal{C}_{L}(b)$ are cones in $\mathcal{X}$ it is called (nonautonomous) cone. The following definition can be found in [KS01], and it is similar to that one in [KS02] where $L$ may depend on $b$. In addition to the definition in [KS02], we use here the auxiliary function $\tilde{L}$ in order to get an estimate in $\left[0, T_{0}\right]$, too.

DEFINITION 2.11. The NDS $\varphi$ satisfies the (nonautonomous) cone invariance property for a cone $\mathcal{C}_{L}$ if there are a function $\left.\tilde{L}:\right] 0, \infty[\rightarrow] 0, \infty\left[\right.$ and a number $T_{0} \geq 0$ such that

$$
\tilde{L}(t) \leq L \quad \text { for } t \geq T_{0}
$$

and such that for $b \in \mathcal{B}$ and $x, y \in \mathcal{X}$,

$$
x-y \in \mathcal{C}_{L}(b)
$$

implies

$$
\varphi(t, b) x-\varphi(t, b) y \in \mathcal{C}_{\tilde{L}(t)}\left(\theta_{t} b\right) \quad \text { for } t>0 .
$$

Now we define a property of a cocycle $\varphi$ which describes a kind of squeezing outside a given cone.

DEFINITION 2.12. The NDS $\varphi$ satisfies the (nonautonomous) squeezing property for a cone $\mathcal{C}_{L}$ if there exist positive constants $K_{1}, K_{2}$ and $\eta$ such that for every $b \in \mathcal{B}, x, y \in \mathcal{X}$ and $T>0$ the identity

$$
\pi_{1}\left(\theta_{T} b\right) \varphi(T, b) x=\pi_{1}\left(\theta_{T} b\right) \varphi(T, b) y
$$


implies for all $x^{\prime} \in \mathcal{X}$ with $\pi_{1}(b) x^{\prime}=\pi_{1}(b) x$ and $x^{\prime}-y \in \mathcal{C}_{L}(b)$ the estimates

$$
\left\|\pi_{i}\left(\theta_{t} b\right)[\varphi(t, b) x-\varphi(t, b) y]\right\|_{\mathcal{Y}} \leq K_{i} \mathrm{e}^{-\eta t}\left\|\pi_{2}(b)\left[x-x^{\prime}\right]\right\|_{\mathcal{Y}}, \quad i=1,2,
$$

for $t \in[0, T]$.

REMARK 2.13. We consider the special case that $\mathcal{B}=\{b\}$ is a singleton. Then the NDS $\varphi$ defines a semiflow $S$ on $\mathcal{X}$ by $S^{t} x=\varphi(t, b) x$ for $(t, x) \in \mathbb{R}_{\geq 0} \times \mathcal{X}$ and we may replace $\pi_{i}(b)$ by $\pi_{i}$. As a special case of the cone invariance property we obtain the

Autonomous cone invariance property: There exists $T_{0} \geq 0$ such that $x, y \in \mathcal{X}$ with

$$
\left\|\pi_{2}[x-y]\right\| \leq L\left\|\pi_{1}[x-y]\right\|
$$

implies

$$
\left\|\pi_{2}\left[S^{t} x-S^{t} y\right]\right\| \leq L\left\|\pi_{1}\left[S^{t} x-S^{t} y\right]\right\| \quad \text { for } t \geq T_{0} .
$$

The squeezing property in the autonomous case becomes the following

Autonomous squeezing property: There exist positive constants $K_{1}, K_{2}, \eta$ such that for every $x, y \in \mathcal{X}$ and $T>0$ the identity

$$
\pi_{1} S^{T} x=\pi_{1} S^{T} y
$$

implies for all $x^{\prime} \in \mathcal{X}$ with $\pi_{1} x^{\prime}=\pi_{1} x$ and $\left\|\pi_{2}\left[x^{\prime}-y\right]\right\| \leq L\left\|\pi_{1}\left[x^{\prime}-y\right]\right\|$ the estimates

$$
\left\|\pi_{i}\left[S^{t} x-S^{t} y\right]\right\| \leq K_{i} \mathrm{e}^{-\eta t}\left\|\pi_{2}\left[x-x^{\prime}\right]\right\|, \quad i=1,2
$$

for $t \in[0, T]$.

Note that our autonomous cone invariance property with $T_{0}=0$ corresponds to the classical cone invariance properties. Our autonomous squeezing property is a modification of the usual squeezing properties, however, at least for semilinear parabolic evolution equations, a strong squeezing property and an additional cone invariance property imply our autonomous squeezing property.

DEFINITION 2.14. The NDS $\varphi$ satisfies the (nonautonomous) boundedness property if for all $t \geq 0$ and all $M_{1} \geq 0$ there exists an $M_{2} \geq 0$ such that for $b \in \mathcal{B}, x \in \mathcal{X}$ with $\left\|\pi_{2}(b) x\right\|_{\mathcal{X}} \leq M_{1}$ the estimate

$$
\left\|\pi_{2}\left(\theta_{t} b\right) \varphi(t, b) x\right\|_{\mathcal{X}} \leq M_{2}
$$

holds.

DEFINITION 2.15. The NDS $\varphi$ satisfies the (nonautonomous) coercivity property if for all $t \geq 0$ and all $M_{3} \geq 0$ there exists an $M_{4} \geq 0$ such that for $b \in \mathcal{B}, x \in \mathcal{X}$ with $\left\|\pi_{1}(b) x\right\|_{\mathcal{X}} \geq M_{4}$ the estimate

$$
\left\|\pi_{1}\left(\theta_{t} b\right) \varphi(t, b) x\right\|_{\mathcal{X}} \geq M_{3}
$$

holds.

The boundedness property ensures that the graph transformation mapping can be defined on a complete metric space of bounded functions. The coercivity property will ensure the existence of global homeomorphisms used for the definition of the graph transformation mapping. 
REMARK 2.16. As we will show later in Section 3.2, for evolution equations the coercivity and boundedness property of $\varphi$ follows from the boundedness of the nonlinearity and exponential dichotomy properties of the linear part. While a global Lipschitz property of the nonlinearity is used for the cone invariance and squeezing property, too, the boundedness of the nonlinearity is an additional restriction.

Therefore, we introduce now another group of technical assumptions which for evolution equations can be verified without boundedness assumption on the nonlinear part.

DEFINITION 2.17. The NDS $\varphi$ satisfies the (nonautonomous) stationarity property if there is a uniformly bounded invariant set $\mathcal{I}$.

The stationarity property together with the cone invariance property allow to define the graph transformation mapping in a space of linearly bounded functions. Obviously it is satisfied, if there is a stationary point.

DEFINITION 2.18. The NDS $\varphi$ satisfies the (nonautonomous) strong coercivity property with respect to invariant set $\mathcal{I}$ and the cone $\mathcal{C}_{L}$ if for all $b \in \mathcal{B}$ there exist positive numbers $M_{5}, M_{6}, M_{7}$ such that for $x \in \mathcal{I}(b)+\mathcal{C}_{L}(b)$ and all $t \geq 0$ the estimate

$$
\left\|\pi_{1}(b) x\right\|_{\mathcal{X}} \leq M_{5} \mathrm{e}^{M_{6} t}\left(M_{7}+\left\|\pi_{1}\left(\theta_{t} b\right) \varphi(t, b) x\right\|_{\mathcal{X}}\right)
$$

holds.

The strong coercivity property ensures the existence of global homeomorphisms used for the definition of the graph transformation mapping and it will be used to show the contractivity of the graph transformation mapping.

REMARK 2.19. As we will show later in Section 3.2, for evolution equations the strong coercivity property of $\varphi$ follows from the uniform boundedness of an invariant set $\mathcal{I}$ and exponential dichotomy properties of the linear part.

If $\mathcal{X} \neq \mathcal{Y}$ we need some properties to compensate the weaker norm.

DEFINITION 2.20. The NDS $\varphi$ satisfies the smoothing property if there are function $\left.M_{8}, M_{9}:\right] 0, \infty[\rightarrow] 0, \infty[$ such that for $x, y \in \mathcal{X}, b \in \mathcal{B}$, and $t>0$ the Lipschitz estimates

$$
\|\varphi(t, b) x-\varphi(t, b) y\|_{\mathcal{X}} \leq M_{8}(t)\|x-y\|_{\mathcal{Y}}
$$

and

$$
\left\|\pi_{1}(b)[x-y]\right\|_{\mathcal{Y}} \leq M_{9}(t)\left\|\pi_{1}\left(\theta_{t} b\right)[\varphi(t, b) x-\varphi(t, b) y]\right\|_{\mathcal{Y}} \quad \text { if } x-y \in \mathcal{C}_{L}
$$

hold.

REMARK 2.21. For parabolic evolution equations this smoothing property is a consequence of global Lipschitz property of the nonlinearity and the smoothing property of parabolic equations.

The following theorem is shown in [KS01], where the boundedness property as required in [KS01] is to weak for the proof in [KS01], and it has to be replaced by the boundedness property as given above. In [KS02] one can find the proof of the following theorem for the special case $\mathcal{X}=\mathcal{Y}$ under the assumption of coercivity and a weaker boundedness property but also with more general cone invariance and squeezing properties (the constant $L$ in the cone invariance property may depend on $b$ ). 
THEOREM 2.22. Let $\varphi$ be an NDS on a Banach space $\mathcal{X} \hookrightarrow \mathcal{Y}$ over a driving system $\theta: \mathbb{R} \times \mathcal{B} \rightarrow \mathcal{B}$ on a set $\mathcal{B}$ and assume that $\varphi$ satisfies the cone invariance and squeezing property.

Moreover, let $\varphi$ satisfy the following technical assumptions:

- $\varphi$ possesses the coercivity and boundedness property or

- $\varphi$ possesses the strong coercivity and stationarity property with respect to the invariant set $\mathcal{I}$ and the cone $\mathcal{C}_{L}$ with a constant $M_{6}<\eta$.

If $\mathcal{X} \neq \mathcal{Y}$, we further assume that

- $\varphi$ possesses the smoothing property, and that $\pi_{1}$ is tempered from above in $\mathcal{X}$, and that there are constants $M_{10}$ and $M_{11}$ with

$$
\left\|\pi_{2}(b)\right\|_{L(\mathcal{X}, \mathcal{X})} \leq M_{10}, \quad\left\|\pi_{1}(b) x\right\|_{\mathcal{Y}} \leq M_{11}\left\|\pi_{1}(b) x\right\|_{\mathcal{X}} \quad \text { for } x \in \mathcal{X}, b \in \mathcal{B} .
$$

Then there exists an inertial manifold $\mathcal{M}=(\mathcal{M}(b))_{b \in \mathcal{B}}$ of $\varphi$ with the following properties:

1. $\mathcal{M}(b)$ is a graph in $\mathcal{X}_{1}(b) \oplus \mathcal{X}_{2}(b)$,

$$
\mathcal{M}(b)=\left\{x_{1}+m\left(b, x_{1}\right): x_{1} \in \mathcal{X}_{1}(b)\right\}
$$

with a mapping $m(b, \cdot)=m(b): \mathcal{X}_{1}(b) \rightarrow \mathcal{X}_{2}(b)$ which is globally Lipschitz continuous

$$
\left\|m\left(b, x_{1}\right)-m\left(b, y_{1}\right)\right\|_{\mathcal{X}} \leq \hat{L}\left\|x_{1}-y_{1}\right\|_{\mathcal{X}}
$$

with some $\hat{L} \geq 0$, and it satisfies

$$
\left\|m\left(b, x_{1}\right)-m\left(b, y_{1}\right)\right\| \mathcal{Y} \leq L\left\|x_{1}-y_{1}\right\|_{\mathcal{Y}}
$$

with $L$ from the cone invariance property.

2. $\mathcal{M}$ is exponentially attracting in $\mathcal{X}$

$$
\left\|\varphi(t, b) x-\varphi(t, b) x^{\prime}\right\|_{\mathcal{X}} \leq \hat{K} \mathrm{e}^{-\eta t}\left\|\pi_{2}(b) x-m\left(b, \pi_{1}(b) x\right)\right\|_{\mathcal{X}}
$$

for $t \geq 1, i=1,2$ with an asymptotic phase $x^{\prime}=x^{\prime}(b, x) \in \mathcal{M}(b)$ of $x$ and some $\hat{K}$ independent of $x, x^{\prime}, b, t$, and we have

$$
\left\|\pi_{i}\left(\theta_{t} b\right)\left[\varphi(t, b) x-\varphi(t, b) x^{\prime}\right]\right\|_{\mathcal{Y}} \leq K_{i} \mathrm{e}^{-\eta t}\left\|\pi_{2}(b) x-m\left(b, \pi_{1}(b) x\right)\right\|_{\mathcal{Y}}
$$

for $t \geq 0, i=1,2$ with $K_{1}, K_{2}>0$ from the squeezing property.

3. If in addition $\pi_{1}$ is tempered from above in $\mathcal{X}$, then $\mathcal{M}$ is pullback attracting in $\mathcal{X}$, more precisely, for each $b \in \mathcal{B}$ there is a $T \geq 0$ such that for each bounded set $\mathcal{D} \subset \mathcal{X}$

$$
d\left(\varphi\left(t, \theta_{-t} b\right) \mathcal{D} \mid \mathcal{M}(b)\right) \leq \mathrm{e}^{-\eta t / 2} d\left(\mathcal{D} \mid \mathcal{M}\left(\theta_{-t} b\right)\right) \quad \text { for } t \geq T .
$$

The constant $T$ can be uniformly chosen for all $b$ belonging to the same $\theta$-orbit. If $\sup _{b \in \mathcal{B}}\left\|\pi_{1}(b)\right\|<\infty$ then $T$ can be chosen independently of $b \in \mathcal{B}$.

3. Inertial manifolds for nonautonomous evolution equations. In the following, we verify the assumptions of Theorem 2.22 for evolution equations under the assumptions of exponential dichotomy conditions on the linear part or under the requirement that the linear part $A$ is constant and selfadjoint such that we may use the eigenvalues of $A$.

Let $\left(\mathcal{X},\|\cdot\|_{\mathcal{X}}\right)$ be a Banach space. 
3.1. Indefinite quadratic forms. Here, let $\mathcal{H}=\mathcal{Z}$ be a Hilbert space equipped with the norm $|\cdot|$, and let $A$ be a (time-independent) densely defined linear operator on $\mathcal{H}$ which is selfadjoint, positive and which has compact resolvent. We consider a nonautonomous parabolic evolution equation

$$
\dot{x}+A x=f(t, x)
$$

where the nonlinear part $f: \mathbb{R} \times \mathcal{X} \rightarrow \mathcal{H}$ satisfies the following assumptions:

- The Hilbert space $\mathcal{X}=D\left(A^{\alpha}\right)$ with norm $\|u\|_{\mathcal{X}}:=|u|_{\alpha}:=\left|A^{\alpha} u\right|$ is the domain of a power $A^{\alpha}$ of $A$ with some $\alpha \in[0,1[$.

- $f(t, x)$ is locally Hölder continuous in $t$ and global Lipschitz continuous in $x$.

Then there are maximally defined (classical) solutions

$$
\mu(\cdot, \tau, \xi) \in C\left(\left[\tau, \infty[, \mathcal{X}) \cap C^{1}(] \tau, \infty[, \mathcal{H})\right.\right.
$$

of (1) with initial condition $x(\tau)=\xi$, see [Hen81, Mik98], and (1) generates a process $\mu$ on $\mathcal{X}$.

Let $\lambda_{1} \leq \lambda_{2} \leq \ldots$ denote the eigenvalues of $A$ counted with their multiplicity and let $e_{1}, e_{2}, \ldots$ denote the corresponding eigenvectors of $A$.

We fix $N \in \mathbb{N}$. Let $\pi_{1}$ be the orthogonal projector from $\mathcal{H}$ onto $\operatorname{span}\left\{e_{1}, \ldots, e_{N}\right\}$ and let $\pi_{2}:=\operatorname{id}_{\mathcal{H}}-\pi_{1}$.

The following lemma is proved in [KS01].

LEMMA 3.1. Under the general assumption given above, let there exist $\rho_{1}<\rho_{2}$, a function $\Lambda:\left[\rho_{1}, \rho_{2}\right] \rightarrow \mathbb{R}$ and a number $\left.\left.L_{0} \in\right] \rho_{1}, \rho_{2}\right]$ with

$$
\Lambda\left(L_{0}\right)>0
$$

and

$$
Q_{\rho}(\mu(t, \tau, x)-\mu(t, \tau, y)) \leq \mathrm{e}^{-2 \Lambda(\rho)(t-\tau)} Q_{\rho}(x-y)
$$

for $\rho \in\left[\rho_{1}, \rho_{2}\right], t \geq \tau, x, y \in \mathcal{X}$ where the quadratic forms $Q_{\rho}: \mathcal{X} \rightarrow \mathbb{R}$ are defined by

$$
Q_{\rho}(x)=\left\|\pi_{2} x\right\|_{\mathcal{Y}}^{2}-\rho^{2}\left\|\pi_{1} x\right\|_{\mathcal{Y}}^{2} \quad \text { for } x \in \mathcal{X}, \quad \rho>0 .
$$

Then the process $\mu$ possesses the cone invariance property for all $L \in\left[\rho_{1}, \rho_{2}\right]$ and the squeezing property with the parameters

$$
L=\rho_{1}, \quad \eta=\Lambda\left(L_{0}\right), \quad K_{1}=\frac{\rho_{2} L_{0}}{\sqrt{\rho_{2}^{2}-L_{0}^{2}} \sqrt{L_{0}^{2}-\rho_{1}^{2}}}, \quad K_{2}=\rho_{1} K_{1} .
$$

REMARK 3.2.

1. The proof of this lemma bases on the derivation of a differential inequality

$$
\frac{\mathrm{d}}{\mathrm{d} t} Q_{\rho}(\mu(t, \tau, x)-\mu(t, \tau, y)) \leq-2 \Lambda(\rho) Q_{\rho}(\mu(t, \tau, x)-\mu(t, \tau, y))
$$

for $t \geq \tau, x, y \in \mathcal{X}$ and $\rho \in\left[\rho_{1}, \rho_{2}\right]$.

2. Obviously, (2) implies the cone invariance property with $L=\rho$ for all $\rho \in\left[\rho_{1}, \rho_{2}\right]$. The squeezing property follows from (2) by combination of inequalities (2) for $\rho=\rho_{1}$ and $\rho=L_{0}$. 
3. In [MPS88], a so-called cone property is used which only requires that

$$
\frac{\mathrm{d}}{\mathrm{d} t} Q_{1}(\mu(t, \tau, x)-\mu(t, \tau, y)) \leq 0
$$

for $t \geq \tau, x, y \in \mathcal{X}$ with $Q_{1}(\mu(t, \tau, x)-\mu(t, \tau, y))=0$. Obviously this condition implies the cone invariance property with $L=1$. Assuming the cone invariance property, the squeezing property can be shown using Gronwall inequalities for the estimation of $\left\|\pi_{2}[\mu(t, \tau, x)-\mu(t, \tau, y)]\right\|_{\mathcal{Y}}$. However, our approach seems to be more general for an extension to time-depending quadratic forms.

Since the exponential dichotomy conditions (8) and additional exponential dichotomy conditions and smoothing properties can be satisfied for $\Phi(t, \tau)=\mathrm{e}^{-A(t-\tau)}$, Theorem 2.22 and Lemmata 2.4, 3.1 imply

THEOREM 3.3. Let the assumptions of Lemma 3.1 be satisfied. Moreover, we assume that

- $f$ is globally bounded or

- there is a bounded invariant set $\mathcal{I}$.

Then the claim of Theorem 2.22 holds for the NDS $\varphi$ generated by (7) with $\eta, L, K_{1}$ and $K_{2}$ as given in Lemma 3.1, i.e., there is an inertial manifold.

REMARK 3.4.

1. An inequality of the form (2) is used in [Rom94] for the special case $\mathcal{Y}=D\left(A^{\alpha / 2}\right)$. Assuming the Lipschitz inequality

$$
|f(x)-f(y)| \leq \ell|x-y|_{\alpha}, \quad x, y \in \mathcal{X}=D\left(A^{\alpha}\right)
$$

and the spectral gap condition

$$
\lambda_{N+1}-\lambda_{N}>\ell\left(\lambda_{N}^{\alpha}+\lambda_{N+1}^{\alpha}\right),
$$

A. V. Romanov [Rom94] shows that (2) holds for $\rho \in\left[h, h^{-1}\right], \Lambda(\rho)=\lambda_{N+1}-\ell \lambda_{N+1}^{\alpha}$ and with $h<1$ satisfying

$$
\lambda_{N+1}-\lambda_{N}>\ell\left(\lambda_{N}^{\alpha}+\frac{1}{2}\left(h^{2}+h^{-2}\right) \lambda_{N+1}^{\alpha}\right) .
$$

Thus our Theorem 3.3 allows to ensure the existence of inertial manifolds under the sharp spectral gap condition (4) for the Lipschitz inequality (3).

2. Let $\mathcal{Y}=\mathcal{H}$ and let $f$ satisfy a Lipschitz inequality of the type

$$
|f(t, x)-f(t, y)| \leq \nu(x-y) \quad \text { for all } x, y \in D(A), \quad t \in \mathbb{R},
$$

where $d_{i}, i=1, \ldots, M$, are positive numbers, $\gamma=\delta_{1} \in\left[0, \min \left\{\alpha, \frac{1}{2}\right\}\right], 0 \leq \delta_{i+1}<\delta_{i}$ for $i=1, \ldots, M-1$ and

$$
\nu(x)=\left(\sum_{i=1}^{M} d_{i}|x|_{\delta_{i}}^{2}\right)^{1 / 2}
$$

Let

$$
g_{1}:=\left(\sum_{i=1}^{M} d_{i} \lambda_{N}^{2 \delta_{i}}\right)^{1 / 2}, \quad g_{2}:=\left(\sum_{i=1}^{M} d_{i} \lambda_{N+1}^{2 \delta_{i}}\right)^{1 / 2}
$$


In [KS01] it is shown that the assumptions of Lemma 3.1 and hence of Theorem 3.3 can be satisfied if the spectral gap condition

$$
\lambda_{N+1}-\lambda_{N}>g_{1}+g_{2}
$$

holds, i.e.

$$
\lambda_{N+1}-\lambda_{N}>\ell\left(\lambda_{N+1}^{\gamma}+\lambda_{N}^{\gamma}\right)
$$

if $\nu(x)=|x|_{\gamma}$.

As a concrete application we consider a reaction-diffusion equation

$$
u_{t}=u_{\xi \xi}+F(\xi, u, \nabla u), \quad u(t, 0)=u(t, 1)=0
$$

with

$$
\left|F(\xi, u, v)-F\left(\xi, u^{\prime}, v^{\prime}\right)\right| \leq \ell_{0}\left|u-u^{\prime}\right|+\ell_{1}\left|v-v^{\prime}\right|
$$

in $\mathcal{Z}=\mathcal{Y}=L_{2}([0,1])$ as studied by P. Brunovský and I. Tereščák [BT91]. Here $-A$ is the Laplacian with Dirichlet boundary condition on $[0,1], f(t, x)(\xi)=F(t, \xi, x(\xi), \nabla x(\xi))$. For the existence theory we need $\alpha>\frac{3}{4}$, but it is possible to choose $\nu(x)=\sqrt{2} \ell_{0}|x|+$ $\sqrt{2} \ell_{1}|x|_{1 / 2}$ in order to cover the gradient in the nonlinearity. Then (6) takes the form

$$
\lambda_{N+1}-\lambda_{N}>\sqrt{2}\left(2 \ell_{0}+\ell_{1}\left(\lambda_{N}^{1 / 2}+\lambda_{N+1}^{1 / 2}\right)\right)
$$

i.e.

$$
(2 N+1) \pi^{2}>2 \sqrt{2} \ell_{0}+\sqrt{2}(2 N+1) \pi \ell_{1}
$$

and is weaker than the spectral gap condition found in [BT91] for the autonomous problem.

3. For some evolution equations it is useful to distinguish the space $\mathcal{X}=D\left(A^{\alpha}\right)$, in which the semiflow acts, from the space $D\left(A^{\gamma}\right)$ used in the Lipschitz inequality: One has to choose $\alpha \in[0,1$ [ in such a way that $f$ is a sufficiently smooth mapping from $\mathbb{R} \times \mathcal{X}$ as required for the existence theory. However it is possible to satisfy and to require a Lipschitz inequality $\|f(x)-f(y)\|_{\mathcal{Z}} \leq \nu(x-y)$ for $x, y \in D(A)$ with $\gamma \in\left[0, \min \left\{\alpha, \frac{1}{2}\right\}[\right.$ and some norm $\nu$ on $D\left(A^{\gamma}\right)$. Especially, for $\nu(x)=\ell|x|_{D\left(A^{\gamma}\right)}$, we find the spectral gap condition

$$
\lambda_{N+1}-\lambda_{N}>\left(\lambda_{N+1}^{\gamma}+\lambda_{N}^{\gamma}\right) \ell
$$

which is weaker than (4) if $\gamma<\alpha$.

3.2. Exponential dichotomy conditions. Let $\mathcal{X} \hookrightarrow \mathcal{Z}$ be Banach spaces equipped with norms $\|\cdot\|_{\mathcal{X}},\|\cdot\|_{\mathcal{Z}}$, and let $(A(t))_{t \in \mathbb{R}}$ be a family of densely defined linear operators $A(t)$ on $\mathcal{Z}$ with domain $D(A(t))$ in $\mathcal{Z}$. We consider a nonautonomous evolution equation

$$
\dot{x}+A(t) x=f(t, x)
$$

which satisfies the following assumptions:

(A1) Linearity $A(t)$ :

- Existence of evolution operator of the linear system: Under suitable additional assumptions on $\mathcal{X}, \mathcal{Z}, A$ and $f$ (see for example [Hen81, DKM92, Lun95]), we may define the evolution operator $\Phi:\left\{(t, s) \in \mathbb{R}^{2}: t \geq s\right\} \rightarrow L(\mathcal{Z}, \mathcal{Z})$ of the linear equation

$$
\dot{x}+A(t) x=0
$$


in $\mathcal{Z}$ as the solution of

$$
\frac{d}{d t} \Phi(t, s)+A(t) \Phi(t, s)=0 \quad \text { for } t>s, s \in \mathbb{R}
$$

and

$$
\Phi(\tau, \tau)=\operatorname{id}_{\mathcal{Z}} \quad \text { for } \tau \in \mathbb{R} .
$$

- There are constants $k_{0}, \ldots, k_{4} \geq 1, \beta_{2}>\beta_{1}, \gamma \in[0,1[$, a monotonously decreasing function $\psi \in C\left(\mathbb{R}_{>0}, \mathbb{R}_{>0}\right)$ with $\psi(t) \leq k_{0} t^{-\gamma}$, and a family $\pi_{1}=\left(\pi_{1}(t)\right)_{t \in \mathbb{R}}$ of linear, invariant projectors $\pi_{1}(t): \mathcal{Z} \rightarrow \mathcal{Z}$, i.e.

$$
\pi_{1}(t) \Phi(t, s)=\Phi(t, s) \pi_{1}(s) \quad \text { for } t \geq s,
$$

such that $\Phi(t, s) \pi_{1}(s)$ can be extended to a linear, bounded operator for $t \in \mathbb{R}$ with

$$
\frac{d}{d t} \Phi(t, s) \pi_{1}(s)+A(t) \Phi(t, s) \pi_{1}(s)=0 \quad \text { for } t, s \in \mathbb{R}
$$

and

$$
\begin{aligned}
& \left\|\Phi(t, s) \pi_{1}(s)\right\|_{L(\mathcal{X}, \mathcal{X})} \leq k_{1} \mathrm{e}^{-\beta_{1}(t-s)} \quad \text { for } t \leq s, \\
& \left\|\Phi(t, s) \pi_{2}(s)\right\|_{L(\mathcal{X}, \mathcal{X})} \leq k_{2} \mathrm{e}^{-\beta_{2}(t-s)} \quad \text { for } t \geq s \text {, } \\
& \left\|\Phi(t, s) \pi_{1}(s)\right\|_{L(\mathcal{Z}, \mathcal{X})} \leq k_{3} \mathrm{e}^{-\beta_{1}(t-s)} \quad \text { for } t \leq s, \\
& \left\|\Phi(t, s) \pi_{2}(s)\right\|_{L(\mathcal{Z}, \mathcal{X})} \leq k_{4} \psi(t-s) \mathrm{e}^{-\beta_{2}(t-s)} \quad \text { for } t>s
\end{aligned}
$$

with $\pi_{2}, \pi_{2}(t)=\operatorname{id}_{\mathcal{Z}}-\pi_{1}(t)$, as the complementary projector to $\pi_{1}$ in $\mathcal{Z}$.

(A2) Nonlinearity $f(t, x)$ : The nonlinear function $f \in C(\mathbb{R} \times \mathcal{X}, \mathcal{Z})$ is assumed to satisfy the Lipschitz inequality

$$
\left\|\pi_{i}(t)[f(t, x)-f(t, y)]\right\|_{\mathcal{Z}} \leq \gamma_{i}\left(\left\|\pi_{1}(t)[x-y]\right\|_{\mathcal{X}},\left\|\pi_{2}(t)[x-y]\right\|_{\mathcal{X}}\right)
$$

for all $t \in \mathbb{R}, x, y \in \mathcal{X}$, where $\gamma_{i}$ are suitable norms on $\mathbb{R}^{2}$.

(A3) Existence of mild solutions: We have the existence and uniqueness of the mild solutions

$$
\mu(\cdot, \tau, \xi) \in C([\tau, \infty[, \mathcal{X})
$$

of (7) with initial condition $x(\tau)=\xi \in \mathcal{X}$, i.e. let $\mu$ be the continuous solution of the integral equation

$$
x(t)=\Phi(t, \tau) \xi+\int_{\tau}^{t} \Phi(t, r) f(\tau, x(r)) \mathrm{d} r \quad \text { for } t \geq \tau .
$$

These were the assumptions.

To include this set-up in the general approach we set $\mathcal{Y}:=\mathcal{X}$.

REMARK 3.5.

1. Exponential dichotomy conditions of the form (8) are used, for example, in [Hen81], [Tem97], [BdMCR98], [LL99], [CS01]. There $k_{3}=\beta_{1}^{\alpha} k_{1}, k_{4}=\beta_{2}^{\alpha}$ with some $\alpha \in[0,1[$ depending on the spaces $\mathcal{X}$ and $\mathcal{Z}$, and $\psi(t)=\beta_{2}^{-\alpha} \max \left\{t^{-\alpha}, 1\right\}, \psi(t)=\beta_{2}^{-\alpha} t^{-\alpha}+1$, or $\psi(t)=\max \left\{\alpha^{\alpha} \beta_{2}^{-\alpha} t^{-\alpha}, 1\right\}$ where $0^{0}:=1$. If $A$ is a time-independent sectorial operator, then usually $\mathcal{X}$ is the domain $D\left((A+a)^{\alpha}\right)$ of the power $(A+a)^{\alpha}$ of $A+a$ with some $\alpha \in[0,1[$ and some $a \in \mathbb{R}$. If $\mathcal{X}=\mathcal{Z}$ then we may choose $\alpha=0$ and $\psi=1$. 
2. In the special case that $A$ is a time-independent, selfadjoint positive linear operator with compact resolvent and dense domain $D(A)$ on the Hilbert space $\mathcal{Z}$, usually one uses $\mathcal{X}=D\left(A^{\alpha}\right)$ with some $\alpha \in\left[0,1\left[\right.\right.$. Let $\pi_{1}$ be the orthogonal projector from $\mathcal{Z}$ onto the linear subspace spanned by the $N$ eigenvectors of $A$ corresponding to the first $N$ eigenvalues $\lambda_{1} \leq \ldots \leq \lambda_{N}$ (counted with their multiplicity). Then we may choose $\beta_{1}=\lambda_{N}, \beta_{2}=\lambda_{N+1}, k_{1}=k_{2}=1, k_{3}=\beta_{1}^{\alpha}, k_{4}=\beta_{2}^{\alpha}, \psi(t):=\max \left\{\alpha^{\alpha} \beta_{2}^{-\alpha} t^{-\alpha}, 1\right\}$, see for example [FST88, Lemma 3.1]. Note that in this case, the approach given in Section 3.1 yields weaker assumption.

3. In [LL99] (and with $\mathcal{X}=\mathcal{Z}$ ), a Lipschitz inequality of the form

$$
\left\|\pi_{i}(t)[f(t, x)-f(t, y)]\right\|_{\mathcal{X}} \leq \ell_{i} \max \left\{\left\|\pi_{1}(t)[x-y]\right\|_{\mathcal{X}},\left\|\pi_{2}(t)[x-y]\right\|_{\mathcal{X}}\right\}
$$

is utilized. This special form of a Lipschitz inequality is contained in our Lipschitz assumption with $\gamma_{i}(w)=\ell_{i}|w|_{\infty}$ and $|\cdot|_{\infty}$ as the maximum norm in $\mathbb{R}^{2}$. The standard Lipschitz inequality

$$
\|f(t, x)-f(t, y)\|_{\mathcal{Z}} \leq \ell\|x-y\|_{\mathcal{X}}
$$

in a Hilbert space $\mathcal{Z}$ and with orthogonal projectors $\pi_{1}(t)$ leads to (9) with $\gamma_{i}(w)=\ell|w|_{2}$ or $\gamma_{i}(w)=\ell|w|_{1}$, where $|\cdot|_{1}$ denotes the sum norm and $|\cdot|_{2}$ denotes the euclidean norm in $\mathbb{R}^{2}$.

4. In [KS01] and under additional exponential dichotomy estimates, the more general situation $\mathcal{X} \hookrightarrow \mathcal{Y} \hookrightarrow \mathcal{Z}$ with

$$
\left\|\pi_{i}(t)[f(t, x)-f(t, y)]\right\|_{\mathcal{Z}} \leq \gamma_{i}\left(\left\|\pi_{1}(t)[x-y]\right\| \mathcal{Y},\left\|\pi_{2}(t)[x-y]\right\|_{\mathcal{Y}}\right)
$$

is considered.

5. If $f$ is globally bounded, then a straight-forward estimation shows that the NDS $\varphi$ generated by (7) possesses the boundedness and coercivity property, see Steps 3 and 4 in the proof of [KS02, Lemma 3.10], and Lemma 3.9 and in [KS01].

6 . If the cone invariance property holds with a globally bounded function $\tilde{L}$ and if $(\mathcal{I}(\tau))_{\tau \in \mathbb{R}}$ is an invariant set which is uniformly bounded in $\tau$, then the NDS $\varphi$ generated by (7) possesses the strong coercivity property, see [KS01, Lemma 3.10].

7. Because of $\mathcal{X}=\mathcal{Y}$, the smoothing property is trivially satisfied.

So the crucial point is to show the cone invariance property and the squeezing property with respect to the projector $\pi_{1}$ for the NDS generated (7).

The main idea used in [KS02, KS01] is to apply comparison theorems in order to estimate the difference of special solutions of (7).

For fixed $r_{1}, r_{2} \geq 0$ and $T \geq 0$, we define

$$
\begin{aligned}
& \left(\Lambda^{1} w\right)(t):=k_{3} \int_{t}^{T} \mathrm{e}^{-\beta_{1}(t-r)} \gamma_{1}(w(r)) \mathrm{d} r \\
& \left(\Lambda^{2} w\right)(t):=k_{4} \int_{0}^{t} \psi(t-r) \mathrm{e}^{-\beta_{2}(t-r)} \gamma_{2}(w(r)) \mathrm{d} r+k_{2} \mathrm{e}^{-\beta_{2} t} L w^{1}(0)
\end{aligned}
$$

and

$$
q(t):=\left(k_{1} \mathrm{e}^{-\beta_{1}(t-T)} r_{1}, k_{2} \mathrm{e}^{-\beta_{2} t} r_{2}\right)
$$


for $t \in[0, T]$ and $w \in C\left([0, T], \mathbb{R}^{2}\right)$. Then $q \in C\left([0, T], \mathbb{R}_{>0}^{2}\right)$. Because of $\psi(t) \leq k_{0} t^{-\gamma}$ with $\gamma \in\left[0,1\left[, \Lambda\right.\right.$ is an at most weakly singular integral operator from $C\left([0, T], \mathbb{R}^{2}\right)$ into $C\left([0, T], \mathbb{R}^{2}\right)$. Moreover, $\Lambda$ is completely continuous.

The next two lemmata are shown in [KS02].

Lemma 3.6. Assume there are $\tilde{L}:] 0, \infty[\rightarrow] 0, \infty\left[, L>0\right.$ and $T_{0} \geq 0$ such that

$$
\tilde{L}(T) \leq L \quad \text { for } T \geq T_{0}
$$

and such that

$$
v^{2}(T) \leq \tilde{L}(T) r_{1}
$$

for each solution $v \in C\left([0, T], \mathbb{R}_{\geq 0}^{2}\right)$ of

$$
v^{i}(t) \leq(\Lambda v)^{i}(t)+q^{i}(t) \quad \text { for } i=1,2, t \in[0, T]
$$

with $r_{1} \geq 0, r_{2}=0$. Then the NDS $\varphi$ generated by (7) possesses the cone invariance property with respect to $\pi$ with the parameters $\tilde{L}, L$ and $T_{0}$.

Lemma 3.7. Assume there are positive numbers $L, \eta, K_{1}, K_{2}$ such that

$$
v^{i}(t) \leq K_{i} \mathrm{e}^{-\eta t} r_{2} \quad \text { for } t \in[0, T]
$$

holds for each $T>0$ and each solution $v \in C\left([0, T], \mathbb{R}_{\geq 0}^{2}\right)$ of (10) with $r_{1}=0, r_{2} \geq 0$. Then $\mu$ possesses the squeezing property with respect to $\pi$ with the parameters $L, \eta$, $K_{1}, K_{2}$.

So it remains to estimate the solutions $v$ of (10).

Let $\mathfrak{B}:=C\left([0, T], \mathbb{R}^{N}\right)$ and let $\mathfrak{C}:=C\left([0, T], \mathbb{R}_{\geq 0}^{N}\right)$. Then $\mathfrak{C}$ is an order cone in $\mathfrak{B}$. The order cone $\mathfrak{C}$ induces a semi-order $\leq \mathfrak{C}$ in $\mathfrak{B}$ by

$$
u \leq_{\mathfrak{C}} w \quad: \Longleftrightarrow w-u \in \mathfrak{C} .
$$

The inequality (10) reads now

$$
v \leq \mathfrak{c} L v+q
$$

and the idea is to estimate $v$ by an upper solution $w$ of the fixed-point problem $u=L u+q$, i.e., by a solution $w \in \mathfrak{C}$ of

$$
L w+q \leq_{\mathfrak{c}} w .
$$

In [KS02, Lemma 3.9] it is shown that the existence of $w^{*} \in \operatorname{int} \mathfrak{C}$ and $\varepsilon \in[0,1[$ with

$$
L w^{*} \leq_{\mathfrak{c}} \varepsilon w^{*}
$$

imply

$$
v \leq w
$$

for any pair $(v, w) \in \mathfrak{C} \times \mathfrak{C}$ of solutions of (11) and (12). So we only have to find a function $w^{*}$ in the interior of $\mathfrak{C}$ with (13) for some $\varepsilon \in[0,1[$ and we have to find solutions $w$ of (12).

For this, one may try to find $w^{*}=w_{2}$ and $w$ as a linear combination of $w_{1}$ and $w_{2}$ where $w_{i}(t):=\mathrm{e}^{-\eta_{i} t}\left(1, \rho_{i}\right)$ with suitable numbers $\eta_{i}$ and $\rho_{i}$. As proved in [KS02], one finds 
Lemma 3.8. Let $t_{*} \geq 0$ be fixed with

$$
\psi_{*}:=\lim _{t \rightarrow t_{*}} \psi(t)<\infty, \quad \psi(t)>\psi_{*} \quad \text { for } t<t_{*} .
$$

Further let

$$
\left.k_{9} \geq \delta \int_{0}^{t_{*}} \psi(r) \mathrm{e}^{-\delta r} \mathrm{~d} r+\psi_{*} \lim _{t \rightarrow t_{*}} \mathrm{e}^{-\delta t} \quad \text { for all } \delta \in\right] 0, \beta_{2}-\beta_{1}[.
$$

Assume that there are positive numbers $\rho_{1}<\rho_{2}$ with

$$
G\left(\rho_{1}\right)=G\left(\rho_{2}\right)=0,\left.\quad G(\rho)\right|_{\left[\rho_{1}, \rho_{2}\right]} \neq 0
$$

and

$$
k_{1} k_{2} \rho_{1}<k_{9}^{-1} \psi_{*} \rho_{2}
$$

where $G: \mathbb{R}_{>0} \rightarrow \mathbb{R}$ is defined by

$$
G(\rho):=\beta_{2}-\beta_{1}-k_{3} \gamma_{1}(1, \rho)-k_{4} k_{9} \rho^{-1} \gamma_{2}(1, \rho) .
$$

Then there are positive numbers $\eta_{1}<\eta_{2}$ with

$$
\eta_{i}=\beta_{1}+k_{3} \gamma_{1}\left(1, \rho_{i}\right)=\beta_{2}-k_{4} k_{9} \rho_{i}^{-1} \gamma_{2}\left(1, \rho_{i}\right),
$$

and the cone invariance and squeezing property hold with

$$
\begin{aligned}
\eta & \left.:=\eta_{2}, \quad L \in\right] k_{1} \rho_{1}, k_{2}^{-1} k_{9}^{-1} \psi_{*} \rho_{2}[ \\
K_{1} & :=\frac{k_{2} k_{9}}{\rho_{2} \psi_{*}-k_{2} k_{9} L}, \quad K_{2}:=\rho_{2} K_{1}
\end{aligned}
$$

and

$$
\tilde{L}(t)=k_{1} \frac{\left(\rho_{2}-\tilde{\rho}\right) \rho_{1} \mathrm{e}^{-\eta_{1} t}+\left(\tilde{\rho}-\rho_{1}\right) \rho_{2} \mathrm{e}^{-\eta_{2} t}}{\left(\rho_{2}-\tilde{\rho}\right) \mathrm{e}^{-\eta_{1} t}+\left(\tilde{\rho}-\rho_{1}\right) \mathrm{e}^{-\eta_{2} t}}
$$

with some $\tilde{\rho} \in] \max \left\{\rho_{1}, k_{2} k_{9} \psi_{*}^{-1} L\right\}, \rho_{2}[$.

Summarizing, we obtain the following theorem as a direct consequence of Theorem 2.22, Lemma 2.4 and Lemma 3.8 .

THEOREM 3.9. Let the assumptions of Lemma 3.8 be satisfied. In addition, let $f$ be globally bounded or let there exist an invariant set $(\mathcal{I}(\tau))_{\tau \in \mathbb{R}}$ on which $f$ is uniformly bounded in $\tau$. Then the claim of Theorem 2.22 holds for the NDS $\varphi$ generated by (7) with $\eta, L, K_{1}$ and $K_{2}$ as given in Lemma 3.8, i.e., there is an inertial manifold.

REMARK 3.10. The crucial point is to verify the assumptions of Lemma 3.8, i.e., to ensure that positive solutions $\rho_{1}<\rho_{2}$ of (16) exist and that they satisfy (17). This condition is a generalization of the well-known spectral gap condition.

1. If

$$
\gamma_{i}(w)=\ell_{i} \max \left\{\left|w^{1}\right|,\left|w^{2}\right|\right\}, \quad \ell_{i}>0 \quad \text { for } i=1,2, \quad w \in \mathbb{R}^{2}
$$

then (17) holds if and only if

$$
\beta_{2}-\beta_{1}>\frac{k_{3} \ell_{1}+k_{4} k_{9} \ell_{2}}{2}+\sqrt{\frac{\left(k_{3} \ell_{1}-k_{4} k_{9} \ell_{2}\right)^{2}}{4}+\frac{k_{1} k_{2} k_{3} k_{4} k_{9}^{2} \ell_{1} \ell_{2}}{\psi_{*}}},
$$


see [KS02, KS01]. If in particular, $k_{1}=k_{2}=k_{3}=k_{4}=1, \alpha=0, \psi=1$, then $k_{9}=\psi_{*}=1$, and we obtain

$$
\beta_{2}-\beta_{1}>\ell_{1}+\ell_{2}
$$

i.e., we obtain the optimal spectral gap condition as found by Y. Latushkin and B. Layton [LL99] for this special form of a Lipschitz condition. A more general situation is considered in $[\mathrm{KS} 02, \mathrm{KS} 01]$.

2. If

$$
\gamma_{i}(w)=\ell_{i 1}\left|w^{1}\right|+\ell_{i 2}\left|w^{2}\right|, \quad \ell_{i 1}, \ell_{i 2}>0 \quad \text { for } i=1,2, \quad w \in \mathbb{R}^{2}
$$

then (17) holds if and only if

$$
\beta_{2}-\beta_{1}>k_{3} \ell_{11}+k_{4} k_{9} \ell_{22}+\frac{k_{1} k_{2} k_{9}+\psi_{*}}{\sqrt{k_{1} k_{2} \psi_{*}}} \sqrt{\ell_{12} \ell_{21} k_{3} k_{4}} .
$$

If, in particular, $k_{3}=k_{1} \beta_{1}^{\alpha}, k_{4}=k_{2} \beta_{2}^{\alpha}, k_{1}=k_{2}=1$, and $\ell_{11}=\ell_{12}=\ell_{21}=\ell_{22}=\ell$, then we obtain the condition

$$
\beta_{2}-\beta_{1}>\left(\beta_{1}^{\alpha}+k_{9} \beta_{2}^{\alpha}+\left(1+k_{9}\right) \sqrt{\beta_{1}^{\alpha} \beta_{2}^{\alpha}}\right) \ell .
$$

For

$$
\psi(t):=\max \left\{\alpha^{\alpha} \beta_{2}^{-\alpha} t^{-\alpha}, 1\right\}
$$

we may choose $t_{*}:=\alpha \beta_{2}^{-1}$ and hence we have $\psi_{*}=1$. Further

$$
k_{10}:=\alpha^{\alpha} \int_{0}^{\alpha} r^{-\alpha} \mathrm{e}^{-r} \mathrm{~d} r+\mathrm{e}^{-\alpha}-1 \geq 0, \quad k_{9}:=1+\frac{\left(\beta_{2}-\beta_{1}\right)^{\alpha}}{\beta_{2}^{\alpha}} k_{10},
$$

and we obtain the condition

$$
\beta_{2}-\beta_{1}>\left(\beta_{1}^{\alpha}+\beta_{2}^{\alpha}+\left(\beta_{2}-\beta_{1}\right)^{\alpha} k_{10}+\left(2+\frac{\left(\beta_{2}-\beta_{1}\right)^{\alpha}}{\beta_{2}^{\alpha}} k_{10}\right) \sqrt{\beta_{1}^{\alpha} \beta_{2}^{\alpha}}\right) \ell .
$$

Now let

$$
\psi(t):=\beta_{2}^{-\alpha} t^{-\alpha}+1
$$

as in [Tem97]. Then $t_{*}=\infty, \psi_{*}=1$, we may choose

$$
k_{10}:=\Gamma(1-\alpha), \quad k_{9}:=1+k_{10},
$$

and we obtain

$$
\beta_{2}-\beta_{1}>\left(\beta_{1}^{\alpha}+\left(2+k_{10}\right) \sqrt{\beta_{1}^{\alpha} \beta_{2}^{\alpha}}+\left(1+k_{10}\right) \beta_{2}^{\alpha}\right) \ell .
$$

This improves the result of [Tem97, Theorem IX.2.1], because our condition is weaker, it does not contain unknown constants and it holds for the nonautonomous case, too.

Finally, let

$$
\psi(t)=\alpha^{\alpha} \beta_{2}^{-\alpha} t^{-\alpha}+1
$$

Then we choose $t_{*}:=\infty$ and have $\psi_{*}=1$. We may choose

$$
k_{10}:=\alpha^{\alpha} \Gamma(1-\alpha), \quad k_{9}:=1+\frac{\left(\beta_{2}-\beta_{1}\right)^{\alpha}}{\beta_{2}^{\alpha}} k_{10}
$$

and we obtain condition (18) but with a little smaller constant $k_{10}$. In particular, we improved the spectral gap conditions found in [BdMCR98] and [CS01], however, here without perturbation and without retardation. 
Summarizing, we believe that our spectral gap conditions are the weakest known spectral gap conditions for (nonautonomous) evolution equations.

\section{References}

[Arn98] L. ARnold, Random Dynamical Systems, Springer, Berlin, 1998.

[BJ89] P. W. BAtes and C. K. R. T. Jones, Invariant manifolds for semilinear partial differential equations, in: Dynamics Reported, Vol. 2, U. Kirchgraber and H.-O. Walther (eds.), Wiley, Chichester, 1989, 1-38.

[BF95] A. Bensoussan and F. Flandoli, Stochastic inertial manifold, Stochastics Stochastics Rep. 53 (1995), 13-39.

[BH96] F. BLOOM and W. HAO, Inertial manifolds of incompressible, nonlinear bipolar viscous fluids, Quart. Appl. Math. 54 (1996), 501-539.

[BdMCR98] L. Boutet De Monvel, I. D. Chueshov, and A. V. Rezounenko, Inertial manifolds for retarded semilinear parabolic equations, Nonlinear Anal. 34 (1998), 907-925.

[BT91] P. Brunovský and I. TereščÁK, Regularity of invariant manifolds, J. Dynam. Differential Equations 3 (1991), 313-337.

[CL99] T. Caraballo and J. A. Langa, Tracking properties of trajectories on random attracting sets, Stochastic Anal. Appl. 17 (1999), 339-358.

[Chu95] I. D. Chueshov, Approximate inertial manifolds of exponential order for semilinear parabolic equations subjected to additive white noise, J. Dynam. Differential Equations 7 (1995), 549-566.

[CS01] I. D. Chueshov and M. Scheutzow, Inertial manifolds and forms for stochastically perturbed retarded semilinear parabolic equations, J. Dynam. Differential Equations 13 (2001), 355-380.

[CFnT86] P. Constantin, C. Foias, B. Nicolaenko, and R. Temam, Nouveaux résultats sur les variétés inertielles pour les équations différentielles dissipatives, C. R. Acad. Sci. Paris Sér. I Math. 302 (1986), 375-378.

[CFnt89a] P. Constantin, C. Foias, B. Nicolaenko, and R. Temam, Integral Manifolds and Inertial Manifolds for Dissipative Partial Differential Equations, Appl. Math. Sci. 70, Springer, New York, 1989.

[CFNT89b] P. Constantin, C. Foias, B. Nicolaenko, and R. Temam, Spectral barriers and inertial manifolds for dissipative partial differential equations, J. Dynam. Differential Equations 1 (1989), 45-73.

[CDF97] H. Crauel, A. Debussche, and F. Flandoli, Random attractors, J. Dynam. Differential Equations 9 (1997), 307-341.

[CF94] H. Crauel and F. Flandoli, Attractors for random dynamical systems, Probab. Theory Related Fields 100 (1994), 365-393.

[Daf71] C. M. Dafermos, An invariance principle for compact processes, J. Differential Equations 9 (1971), 239-252; erratum: ibid. 10 (1971), 179-180.

[DKM92] D. Daners and P. Koch Medina, Abstract Evolution Equations, Periodic Problems and Application, Pitman Res. Notes Math. Ser. 279, Longman Scientific \& Technical, Harlow, 1992.

[DG91] F. Demengel and J.-M. Ghidaglia, Some remarks on the smoothness of inertial manifolds, Nonlinear Anal. 16 (1991), 79-87.

[DLS01] J. DUAN, K. Lu, and B. Schmalfuss, Invariant manifolds for stochastic partial differential equations, Ann. Probab. (to appear). 
[FS96] F. Flandoli and B. Schmalfuss, Random attractors for the 3D stochastic NavierStokes equation with multiplicative white noise, Stochastics Stochastics Rep. 59 (1996), 21-45.

[FnSt85] C. Foias, B. Nicolaenko, G. R. Sell, and R. Temam, Variétés inertielles pour l'équation de Kuramoto-Sivashinsky, C. R. Acad. Sci. Paris Sér. I Math. 301 (1985), $285-288$.

[FNST88] C. Foias, B. Nicolaenko, G. R. Sell, and R. Temam, Inertial manifolds for the Kuramoto-Sivashinsky equation and an estimate of their lowest dimension, J. Math. Pures Appl. (9) 67 (1988), 197-226.

[FST85] C. Foias, G. R. Sell, and R. Temam, Variétés inertielles des équations différentielles dissipatives, C. R. Acad. Sci. Paris Sér. I Math. 301 (1985), 139-141.

[FST88] C. Foias, G. R. Sell, and R. Temam, Inertial manifolds for nonlinear evolutionary equations, J. Differential Equations 73 (1988), 309-353.

[FST89] C. FoiAs, G. R. Sell, and E. S. Titi, Exponential tracking and approximation of inertial manifolds for dissipative nonlinear equations, J. Dynam. Differential Equations 1 (1989), 199-244.

[GV97] A. YU. GoritskiI and M. I. Vishik, Local integral manifolds for a nonautonomous parabolic equation, Tr. Semin. im. I. G. Petrovskogo No. 19 (1996), 304-322, 348-349 (in Russian); English transl.: J. Math. Sci. (New York) 85 (1997), 2428-2439.

[Had01] J. HadAmard, Sur l'iteration et les solutions asymptotiques des équations différentielles, Bull. Soc. Math. France 29 (1901), 224-228.

[Hal80] J. K. Hale, Ordinary Differential Equations, second ed., Robert E. Krieger Publishing Co., Inc., Huntington, 1980.

[HR92] J. K. HALE and G. RAUGEL, Reaction-diffusion equation on thin domains, J. Math. Pures Appl. (9) 71 (1992), 33-95.

[Hen81] D. Henry, Geometric Theory of Semilinear Parabolic Equations, Lecture Notes in Math. 840, Springer, Berlin, 1981.

[JT96] D. A. Jones and E. S. Tiтi, $C^{1}$ approximations of inertial manifolds for dissipative nonlinear equations, J. Differential Equations 127 (1996), 54-86.

[Kam84] D. A. KAmAev, Hopf's conjecture for a class of chemical kinetics equations, Zap. Nauchn. Sem. Leningrad. Otdel. Mat. Inst. Steklov. (LOMI) 110 (1981), 57-73, 242 (in Russian); English transl.: J. Soviet Math. 25 (1984), 836-849.

[KS97a] P. E. Kloeden and B. Schmalfuss, Cocycle attractors of variable time-step discretizations of Lorenzian systems, J. Differ. Equations Appl. 3 (1997), 125-145.

[KS97b] P. E. Kloeden and B. Schmalfuss, Nonautonomous systems, cocycle attractors and variable time-step discretization, Numer. Algorithms 14 (1997), 141-152.

[KS98] P. E. KLOEDEN and D. J. STONIER, Cocycle attractors in nonautonomously perturbed differential equations, Dynam. Contin. Discrete Impuls. Systems 4 (1998), 211-226.

[KS01] N. KoKSCH and S. SiEgmund, Inertial manifolds for nonautonomous dynamical systems and for nonautonomous dynamical systems, CD ROM Proceedings of the Equadiff 10, Prague, 2001.

[KS02] N. KoKSCH and S. Siegmund, Pullback attracting inertial manifolds for nonautonomous dynamical systems, J. Dynam. Differential Equations 14 (2002), 889-941.

[Kra68] M. A. KRAsnoseL'skII, The Operator of Translation along Trajectories of Differential Equations, Transl. Math. Monogr. 19, Amer. Math. Soc., Providence, 1968.

[LL99] Y. LATUSHKIN and B. LAYTON, The optimal gap condition for invariant manifolds, Discrete Contin. Dynam. Systems 5 (1999), 233-268. 
[Lun95] A. LunARd, Analytic Semigroups and Optimal Regularity in Parabolic Problems, Progr. Nonlinear Differential Equations Appl. 16, Birkhäuser, Basel, 1995.

[LS89] M. Luskin and G. R. SELL, Approximation theories for inertial manifolds, RAIRO Modél. Math. Anal. Numér. 23 (1989), 445-461.

[Lya47] A. M. Lyapunov, Problème gènèral de la stabilité de mouvement (translation of the Russian edition originally published in 1892 by the Mathematics Society of Kharkov), Ann. of Math. Stud. 17, Princeton University Press, Princeton, 1947.

[Lya92] A. M. Lyapunov, The general problem of stability of motion. With a biography of Lyapunov by V. I. Smirnov and a bibliography of Lyapunov's work by J. F. Barrett, transl. and ed. by A. T. Fuller, Taylor \& Francis, London, 1992.

[MPS88] J. Mallet-Paret and G. R. Sell, Inertial manifolds for reaction diffusion equations in higher space dimensions, J. Amer. Math. Soc. 1 (1988), 805-866.

[Man77] R. MAÑÉ, Reduction of semilinear parabolic equations to finite dimensional $C^{1}$ flows, in: Geometry and Topology, J. Palis and M. do Carmo (eds.), Lecture Notes in Math. 597, Springer, Berlin, 1977, 361-378.

[MSZ00] A. Mielke, G. Schneider, and A. Ziegra, Comparison of inertial manifolds and application to modulated systems, Math. Nachr. 214 (2000), 53-69.

[Mik98] M. MiklavČıč, Applied Functional Analysis and Partial Differential Equations, World Scientific, River Edge, 1998.

[Mor83] X. MorA, Finite dimensional attracting manifolds in reaction-diffusion equations, in: Nonlinear Partial Differential Equations (Durham, 1982), J. A. Smoller (ed.), Contemp. Math. 17, Amer. Math. Soc., Providence, 1983, 353-360.

[Nin93] H. NinomiYA, Inertial manifolds and squeezing properties, in: Nonlinear PDEJAPAN Symposium 2 (Kyoto, 1991), K. Masuda, M. Mimura and T. Nishida (eds.), Lect. Notes Numer. Appl. Anal. 12, Kinokuniya, Tokyo, 1993, 175-202.

[Per28] O. Perron, Über Stabilität und asymptotisches Verhalten der Integrale von Differentialgleichungssystemen, Math. Z. 29 (1928), 129-160.

[Per29] O. Perron, Über Stabilität und asymptotisches Verhalten der Lösungen eines Systems endlicher Differenzengleichungen, J. Reine Angew. Math. 161 (1929), 41-64.

[Per30] O. Perron, Die Stabilitätsfrage bei Differentialgleichungen, Math. Z. 32 (1930), 703-728.

[Rob93] J. C. Robinson, Inertial manifolds and the cone condition, Dynam. Systems Appl. 2 (1993), 311-330; erratum: ibid. 3 (1994), 451.

[Rob96] J. C. RoBinson, The asymptotic completeness of inertial manifolds, Nonlinearity 9 (1996), 1325-1340.

[Rob01] J. C. Robinson, Infinite-Dimensional Dynamical Systems. An Introduction to Dissipative Parabolic PDEs and the Theory of Global Attractors, Cambridge Texts Appl. Math., Cambridge Univ. Press, Cambridge, 2001.

[RB95] A. Rodríguez-Bernal, Attractors and inertial manifolds for the dynamics of a closed thermosyphon, J. Math. Anal. Appl. 193 (1995), 942-965.

[Rom94] A. V. Romanov, Sharp estimates of the dimension of inertial manifolds for nonlinear parabolic equations, Izv. Ross. Akad. Nauk Ser. Mat. 57 (1993), no. 4, 36-54 (in Russian); English transl.: Russ. Acad. Sci. Izv. Math. 43 (1994), 31-47.

[SK95] H. SANo and N. Kunimatsu, An application of inertial manifold theory to boundary stabilization of semilinear diffusion systems, J. Math. Anal. Appl. 196 (1995), $18-42$.

[Sch92] B. Schmalfuss, The stochastic attractor of the stochastic Lorenz system, in: Nonlinear Dynamics: Attractor Approximation and Global Behaviour (ISAM '92), N. Koksch, V. Reitmann, and T. Riedrich (eds.), Technical University Dresden, 1992, 27-52. 
[SY92] G. R. SELL and Y. You, Inertial manifolds: the non-self-adjoint case, J. Differential Equations 96 (1992), 203-255.

[SY02] G. R. Sell and Y. You, Dynamics of Evolutionary Equations, Appl. Math. Sci. 143, Springer, New York, 2002.

[TY94] M. TABOADA and Y. YOU, Invariant manifolds for retarded semilinear wave equations, J. Differential Equations 114 (1994), 337-369.

[Tem88] R. Temam, Infinite-Dimensional Dynamical Systems in Mechanics and Physics, Appl. Math. Sci. 68, Springer, New York, 1988.

[Tem97] R. Temam, Infinite-Dimensional Dynamical Systems in Mechanics and Physics, 2nd ed., Appl. Math. Sci. 68, Springer, New York, 1997.

[WF97] Z. WANG and X. FAN, Inertial manifolds for non-autonomous evolution equations, Adv. in Math. (China) 26 (1997), 185-186. 